\title{
A Retrospective Data Review Confirms That Topical Preservative-Free Hydrocortisone Improves Inflammation in Dry Eye Disease
}

This article was published in the following Dove Press journal:

Clinical Ophthalmology

\author{
Biljana Kuzmanović Elabjer (D) \\ Leon Marković \\ Mirjana Bjeloš \\ Mladen Bušić \\ Daliborka Miletić \\ Eva Kos
}

University Eye Clinic, Faculty of Dental Medicine and Health Care Osijek, Faculty of Medicine Osijek, University Josip Juraj Strossmayer in Osijek, University Hospital "Sveti Duh", Zagreb, Croatia
Correspondence: Mladen Bušić University Eye Clinic, Faculty of Dental Medicine and Health Care Osijek, Faculty of Medicine Osijek, University Josip Juraj Strossmayer in Osijek, University Hospital "Sveti Duh", WHO Collaborating Centre for Child Eye Care, Sveti Duh 64, Zagreb 10000 , Croatia

Tel +3859/37/2167

Email mbusic@kbsd.hr
Background: Inflammation is among the most important mechanisms in the pathogenesis of dry eye disease (DED), triggering the vicious circle of the disease. Reducing inflammation is an important target in dry eye disease treatment. Hydrocortisone is a low-potency corticosteroid with a low ocular penetration potential.

Aim: To document the effect of topical preservative-free hydrocortisone $0.335 \%$ ( $\mathrm{PFH}$, Softacort $^{\circledR}$, Laboratoires Théa, France) on DED.

Methods: Retrospective data review of patients with mild to moderate DED, treated with PFH for 15 days. Clinical evaluations at Days 0 and 15 included the assessment of the central precorneal tear film thickness (CPTFT), fluorescein tear breakup time, Schirmer test, corneal grading staining (Oxford schema), ocular surface disease index (OSDI) spatial distribution of the precorneal tear film thickness, intraocular pressure (IOP) and local tolerance.

Results: Data from 13 women and 2 men were collected. Mean age \pm SD was $51 \pm 5$ years for women and $53 \pm 4$ years for men. Clinical signs and symptoms significantly (all $\mathrm{p}<0.05$ ) improved after 15 days of treatment. A significant positive correlation between the percentage of change in left eye CPTFT and that in the contralateral eye CPTFT was observed $(p=0.003)$ as well as for both eyes and the left eye FTBUT $(p=0.03)$. For the percentage of change in OSDI, the only significant correlation was with the percentage of change in right eye and FTBUT $(\mathrm{p}=0.03$ ). IOP remained unchanged. No adverse events were recorded.

Conclusion: This retrospective data review confirms that topical PFH twice daily for 2 weeks significantly improves clinical signs and symptoms in patients with mild to moderate DED with no safety issues.

Keywords: dry eye disease, inflammation, topical preservative-free hydrocortisone, corticosteroids, central precorneal tear film, ocular surface disease index

\section{Introduction}

Ocular surface inflammation plays an important etiological role in dry eye disease (DED). ${ }^{1}$ Osmotic stress activates mitogen-activated protein kinase (MAPK) and nuclear factor (NF) $-\kappa B$ in the ocular surface epithelium (OSE). Moreover, it triggers the production of pro-inflammatory cytokines and chemokines IL- $1 \beta$, TNF- $\alpha$, and IL- 8 as well as matrix metalloproteinase $-1,-3,-9,-10$, and -13 and up-regulates major histocompatibility complex class II on the OSE, mobilizes and activates antigenpresenting cells, recruits Th1 and Th17 cells to the inflammatory site and finally increases the immune response, resulting in a vicious circle of tissue destruction and tear film damage. ${ }^{2-6}$ 
Artificial tears are the basics to treat DED while antiinflammatory treatment is mandatory to interrupt the vicious circle of surface damage and inflammation. ${ }^{6,7}$ Anti-inflammatory treatments include topical cyclosporine, corticosteroids, tacrolimus/pimecrolimus, tetracyclines, lifitegrast and macrolides with Corticosteroids being the main treatment in inflammatory DED. ${ }^{7-9}$ Hydrocortisone is a low-potency corticosteroid with a low ocular penetration potential. ${ }^{10,11}$ Thus, intraocular adverse effects are minimized, especially the increase of intraocular pressure (IOP). ${ }^{12}$

Recently, a post-marketing clinical study confirmed the efficacy of topical preservative-free hydrocortisone $0.335 \%$ (PFH, Softacort ${ }^{\circledR}$, Laboratoires Théa, France) in the treatment of inflammation in patients with DED. ${ }^{13}$

This article reports results from a retrospective data review of patients with mild to moderate DED, treated with $\mathrm{PFH}$, in order to document its effect on inflammation in DED.

\section{Methods}

Records of 15 consecutive patients with mild to moderate DED (13-32 points) according to the ocular surface disease questioning (OSDI scoring tool), treated twice daily with topical PFH $0.335 \%$ were collected between February 2019 and March 2019. ${ }^{14}$ The study complied with the requirements of the Declaration of Helsinki, national requirements for the conduct of retrospective studies and received approval from the University Josip Juraj Strossmayer research ethics committee on 28 March 2019. No informed consent form was to be obtained according to local legal requirements for retrospective studies. However, patients for whom data were reviewed received an information sheet confirming data protection.

All patients used concomitantly preservative-free lubricants with sodium hyaluronate as an active ingredient. Patients were not allowed to use topical products containing trehalose or hyaluronic acid, did not suffer from noncicatricial or cicatricial Meibomian gland dysfunction, or another anterior segment co-morbidity that required topical therapy (such as cyclosporine, other anti-inflammatory agents, glaucoma therapy), did not have a history of surgically induced dry eye (eg, after refractive surgery), did not wear contact lenses, did not suffer from lagophthalmos, Graves orbitopathy, or systemic autoimmune diseases treated with topical and/or systemic anti-inflammatory therapy, and/or were not taking systemic drugs known to reduce tear production.
We collected data from 2 visits: one prior to treatment (Day 0) and one 15 days later (Day 15). On both occasions, a clinical eye examination and the following tests were performed: corneal grading staining carried out according to the Oxford schema using fluorescein strip (Optitech ${ }^{\circledR}$, London, UK), anterior segment photography (BX 900 Photo Slit Lamp, Haag-Streit, Switzerland), fluorescein tear breakup time (FTBUT), Schirmer test I, pachymetry of the central precorneal tear film thickness (CPTFT) and spatial distribution of the precorneal tear film thickness on a pachymetry map using fluorescein performed with a Pentacam-rotating Scheimpflug camera (version 1.21r43, Pentacam Oculus Systems, Wetzlar, Germany), applanation tonometry to assess intraocular pressure (IOP) and OSDI scoring. After corneal thickness was measured, a fluorescein strip containing $1 \mathrm{mg}$ of fluorescein sodium was wetted with $0.05 \mathrm{~mL}$ (one drop) of sterile saline. The fluorescein strip was then applied to the inferior tear meniscus while the patient was instructed to look upward. Immediately after fluorescein instillation, patients had their eyes closed for one minute. Corneal thickness was measured again using a blue light source. The difference in central corneal thickness prior to and after fluorescein instillation was considered as the central precorneal tear film thickness. According to the spatial distribution of the precorneal tear film thickness on the pachymetry map, patients were classified into 3 patterns: pattern 1 was described as thickening of the precorneal tear film upward from the cornea; pattern 2, as uniform distribution; and pattern 3 , as thickening of the precorneal tear film downward from the cornea. ${ }^{15}$

The following ophthalmological examinations were performed by the same investigator: (1) central precorneal tear film, measured in $\mu \mathrm{m}$; (2) fluorescein tear breakup time, recorded in seconds; (3) Schirmer test, measured in $\mathrm{mm}$; (4) corneal grading staining carried out according to the Oxford schema; (5) ocular surface disease index (OSDI); (6) spatial distribution of the precorneal tear film thickness on a pachymetry map and (7) IOP. Data obtained prior to PFH therapy on Day 0 and on Day 15 were analyzed for both eyes for each outcome.

Descriptive statistics, regression analysis and Pearson product-moment correlation coefficient were performed using MathCalc ver 2.1 statistical analysis purposes. A p-value $<0.05$ was considered statistically significant.

\section{Results}

Overall, data from 13 women and 2 men were collected. Mean age \pm SD was $51 \pm 5$ years for women and $53 \pm 4$ years for men. Details about DED data at Day 0 are given in Table 1. 
Table I Patient Demographic and DED Data at Day 0I (Mean Values)

\begin{tabular}{|l|l|}
\hline Parameters & Value \\
\hline $\begin{array}{l}\text { Number of patients }(\mathrm{n}, \%) \\
\text { Women }\end{array}$ & $13(8.66 \%)$ \\
Men & $2(13.33 \%)$ \\
\hline Age (Mean \pm SD, years) & \\
Women & $5 \mathrm{I} \pm 5$ years \\
Man & $53 \pm 4$ years \\
\hline CPTFT (Mean \pm SD, $\mu \mathrm{m})$ & \\
Right eye & $20.93 \pm 5.0 \mathrm{I}$ \\
Left eye & $20.4 \pm 4.22$ \\
\hline FTBUT (Mean $\pm S D$, sec) & \\
Right eye & $4.33 \pm 1.5$ \\
Left eye & $4.33 \pm 1.18$ \\
\hline Schirmer test (Mean $\pm S D, \mathrm{~mm})$ & \\
Right eye & $8.26 \pm 3.8 \mathrm{I}$ \\
Left eye & $8.4 \pm 4.38$ \\
OSDI score & $24.53 \pm 4.39$ \\
\hline Corneal grading (Mean $\pm S D$, Oxford schema) & \\
Right eye & $2.8 \pm 0.94$ \\
Left eye & $3.0 \pm 0.65$ \\
Overall IOP (Mean $\pm S D, m m H g)$ & $15.0+2.0$ \\
\hline
\end{tabular}

Abbreviations: CPTFT, central precorneal tear film; FTBUT, fluorescein tea breakup; OSDI, ocular surface disease index, SD, standard deviation, sec, seconds.

The mean change before instillation of PFH from Day 0 at Day 15 for CPTFT was $18.71 \pm 25.18 \mu \mathrm{m}$ for the right $(\mathrm{p}=0.000015)$ and $15.07 \pm 21.44(\mathrm{p}=0.0003) \mu \mathrm{m}$ for the left eye. The mean change for FTBUT in the right eye was 61.27 \pm 38.74 ( $\mathrm{p}=0.009)$ seconds and $55.68 \pm 42.03$ seconds $(\mathrm{p}=0.08)$ in the left eye. The Schirmer test showed a mean percent improvement of $77.97 \pm 11.35 \%$ for the right $(\mathrm{p}=0.03)$ and $63.13 \pm 67.75 \%(\mathrm{p}=0.002)$ for the left eye. Mean OSDI scores had significantly $(\mathrm{p}=0.001)$ decreased by $31.79 \pm 4.53 \%$, corresponding to a reduction of 7.8 points.

However, no statistically significant improvement for corneal staining was observed using the Oxford schema ( $40.48 \pm 11.20$ for the right and $40.0 \pm 14.37$ for the left eye).

A negative value in CPTFT change was found in 2 patients, for each in one single eye. A negative change in Schirmer test value was documented in one patient. An increase in OSDI score was also observed in one patient.

Figure 1 provides data for Day 0 and Day 15 for CPTFT, FTBUT, Schirmer test, OSDI scores and corneal staining.

Based on CPTFT on Day 0, patients were divided into 2 groups: those with a CPTFT $<24 \mu \mathrm{m}$ and those with a CPTFT $\geq 24 \mu \mathrm{m}$; the mean percent change in CPTFT was evaluated. In the group with a CPTFT $\leq 24 \mu \mathrm{m}$ on Day 0 , the mean percent change in CPTFT was significantly higher; conversely, in patients with a CPTFT $\geq 24 \mu \mathrm{m}$ on Day 0, CPTFT either stayed unchanged or decreased (Figure 2).

On Day 0 , according to the pachymetry maps, pattern 1 was documented in 13/30 eyes, pattern 2 in 9/30 eyes, and pattern 3 in $8 / 30$ eyes. This spatial distribution remained almost unchanged after 15 days.

Correlation coefficients (CC) obtained by comparing the mean percentages of change, for the various outcomes, are shown in Table 2. According to the CPTFT, Schirmer test, and corneal grading staining carried out according to the Oxford schema, there was a significant correlation between the percent change observed in one eye and in the other eye in a same patient. A significant positive correlation between the percentage of change in left eye CPTFT and that in the contralateral eye CPTFT was observed $(p=0.003)$ as well as for both eyes and the left eye FTBUT $(p=0.03)$. For the percentage of change in OSDI, the only significant correlation was with the percentage of change in right eye FTBUT $(\mathrm{p}=0.03)$.

IOP remained stable $(14.0 \pm 3.0 \mathrm{mmHg}$ at Day $15 \mathrm{vs}$ $15.0 \pm 2.0 \mathrm{mmHg}$ at Day 0) in all patients; no adverse events were recorded.

\section{Discussion and Conclusion}

To the best of our knowledge, this is the first report of a significant improvement in mean central precorneal tear film thickness, OSDI, and Schirmer test after 2 weeks of ocular instillation of PFH twice daily in patients with mild DED. The present results on mean central precorneal tear film thickness $(20.93 \pm 5.01 \mu \mathrm{m}$ for the right eye and 20.40 $\pm 4.22 \mu \mathrm{m}$ for the left eye) in patients with non-treated DED are in agreement with those published earlier on tear film thickness in humans. ${ }^{15,16}$ By modifying the technique of fluorescein application, we generated a simple way to measure precorneal tear film thickness, using the Pentacam-rotating Scheimpflug camera.

To assess the effect of hydrocortisone, the study excluded patients using products containing trehalose and hyaluronic acid, because of their effects on ocular surface inflammatory markers. ${ }^{17}$ The current therapeutic indication of PFH is mild non-infectious inflammatory conjunctival diseases. ${ }^{18}$ Thus, we only were able to recruit 15 patients with mild DED for this study. However, the gender ratio of the study population represented the real gender ratio in 


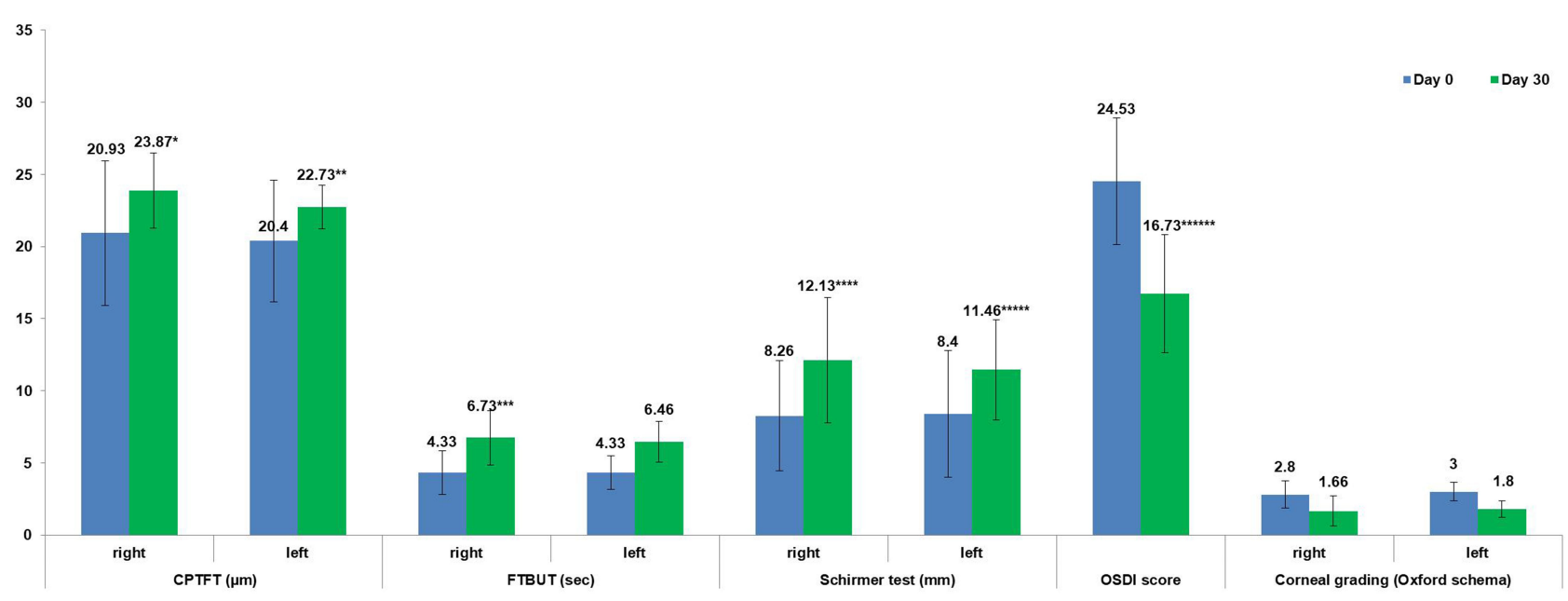

Figure I Mean results for central precorneal tear film thickness, fluorescein tear breakup time, Schirmer test, ocular surface disease index and corneal grading at Day 0 and Day 15. * ${ }^{*}=0.000015, * * p=0.0003, * * * p=0.009, * * * * p=0.03$, ***** $p=0.002, * * * * * * p=0.001$.

Abbreviations: CPTFT, central precorneal tear film thickness; FTBUT, fluorescein tear breakup time; OSDI, ocular surface disease index; corneal grading staining done by Oxford schema; sec, second.

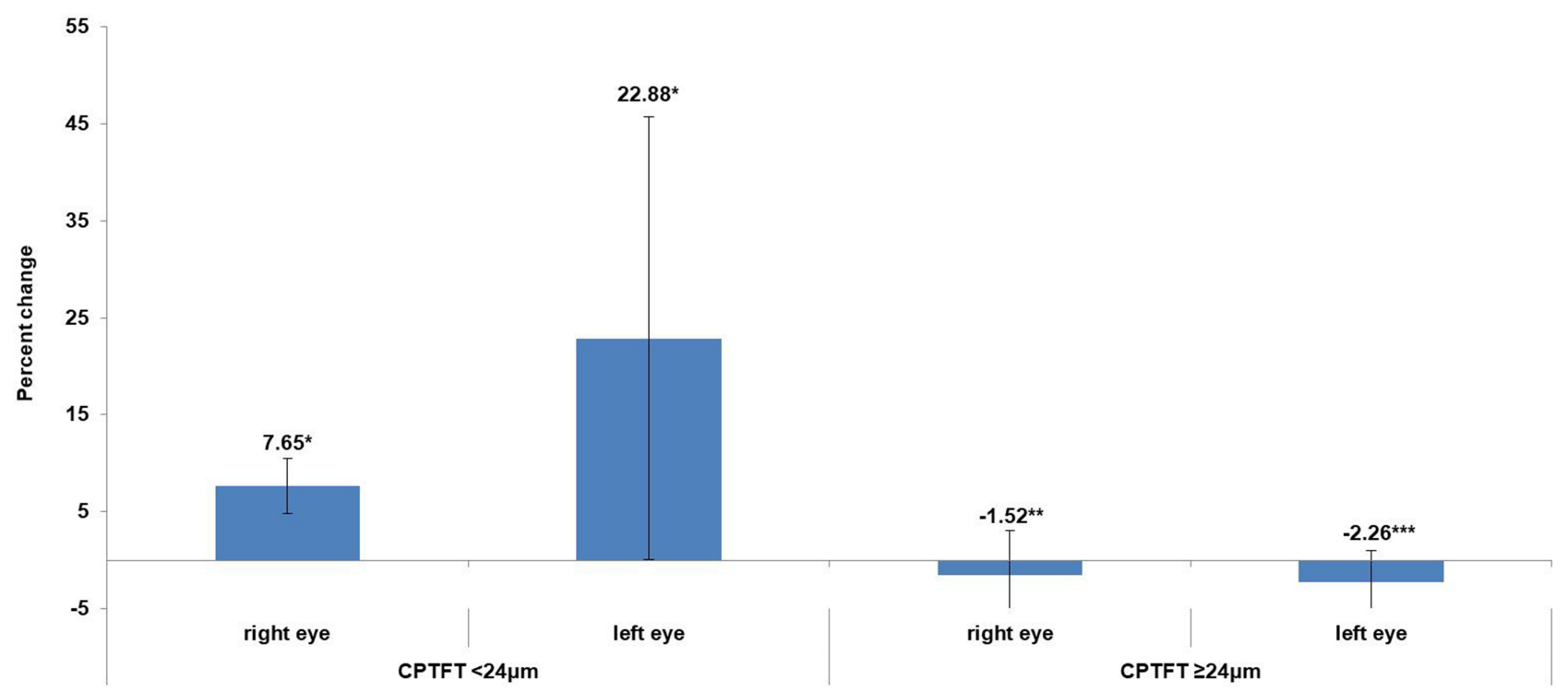

Figure 2 Mean percent change in tear film thickness between Day 0 and Day 15, according to CPTFT $(<24 \mu \mathrm{m}$ or $\geq 24 \mu \mathrm{m})$ at Day 0. ${ }^{*} \mathrm{p}=0.004 . * * \mathrm{*}=0.03$. *** $\mathrm{p}=0.02$.

DED well. ${ }^{19}$ In addition, the mean age of the study patients fits the criterion for higher risk of DED. ${ }^{20}$

Pachymetry of the precorneal tear film using fluorescein with the Pentacam-rotating Scheimpflug camera was first described by Zhuang et al. ${ }^{15}$ In the present study, a novel technique for applying fluorescein to the eye was introduced. Based on a non-published pilot study on 5 patients, one minute of eye closure after fluorescein instillation led to the stabilization of the tear film thickness, with an intra-observer reliability of $80 \%$.

Tear film thickness is determined by numerous variable factors. A previous study on precorneal tear film thickness yielded inconsistent results, ranging from 3 to $40 \mu \mathrm{m} .{ }^{15}$ Only a scarce amount of data exists regarding the difference in tear film thickness between normal and dry eye. ${ }^{15}$ To date, no data have been published in literature regarding the change in tear film thickness measured with a Scheimpflug camera after topical corticosteroid treatment. In the present study, PFH led to a significant increase in CPTFT measured with a Scheimpflug camera. The positive correlation between the percentage of change in left eye CPTFT and that in the contralateral eye was significant, as was the correlation between the percentage of change in CPTFT in both eyes and that in left eye 
Table 2 Correlation Coefficients of Percentages of Change in Tear Film Thickness Between the Different Tests, and p-values

\begin{tabular}{|l|l|l|l|l|l|l|l|l|l|}
\hline & R- CPTFT & L- CPTFT & R-FTBUT & L-FTBUT & R-Sc & L-Sc & OSDI & R-OX & L-OX \\
\hline R-CPTFT & 1.0 & $0.78(0.0003)$ & $0.47(0.06)$ & $0.69(0.03)$ & $-0.2(0.9)$ & $-0.47(0.06)$ & $0.26(0.32)$ & $0.25(0.33)$ & $0.16(0.52)$ \\
L-CPTFT & $0.78(0.0003)$ & 1.0 & $0.16(0.55)$ & $0.68(0.03)$ & $-0.24(0.35)$ & $-0.37(0.14)$ & $0.3(0.26)$ & $0.25(0.34)$ & $0.24(0.36)$ \\
R-FTBUT & $0.47(0.06)$ & $0.16(0.55)$ & 1.0 & $0.23(0.37)$ & $0.45(0.07)$ & $0.07(0.77)$ & $0.54(0.03)$ & $0.42 .(0.09)$ & $0.26(0.33)$ \\
L-FTBUT & $0.69(0.03)$ & $0.68(0.03)$ & $0.23(0.37)$ & 1.0 & $-0.14 .(0.59)$ & $-0.13(0.62)$ & $0.32(0.21)$ & $0.42(0.09)$ & $0.40(0.12)$ \\
R-Sc & $-0.2(0.9)$ & $-0.24(0.35)$ & $0.45(0.07)$ & $-0.14(0.59)$ & 1.0 & $0.62(0.09)$ & $0.02(0.9)$ & $0.26(0.32)$ & $0.03(0.89)$ \\
L-Sc & $-0.47(0.06)$ & $-0.37(0.14)$ & $0.07(0.77)$ & $-0.13(0.62)$ & $0.62(0.09)$ & 1.0 & $0.11(0.67)$ & $0.23(0.38)$ & $0.18(0.50)$ \\
OSDI & $0.26(0.32)$ & $0.3(0.26)$ & $0.54 .(0.03)$ & $0.32(0.21)$ & $0.02(0.9)$ & $0.11(0.67)$ & 1.0 & $0.35(0.17)$ & $0.1(0.68)$ \\
R-OX & $0.25(0.33)$ & $0.25(0.34)$ & $0.42(0.09)$ & $0.42(0.09)$ & $0.26(0.32)$ & $0.23 .(0.38)$ & $0.35(0.17)$ & 1.0 & $0.69(0.03)$ \\
L-OX & $0.16(0.52)$ & $0.24(0.36)$ & $0.26(0.33)$ & $0.40(0.12)$ & $0.03(0.89)$ & $0.18(0.50)$ & $0.1(0.68)$ & $0.69(0.03)$ & 1.0 \\
\hline
\end{tabular}

Abbreviations: R-CPTFT, right eye central precorneal tear film; L-CPTFT, left eye central precorneal tear film thickness; R-FTBUT, right eye fluorescein tear breakup; L-FTBUT, left eye fluorescein tear breakup time; R-Sc, right eye Schirmer test; L-Sc, left eye Schirmer test; OSDI, ocular surface disease index; R-Ox, corneal grading staining done by Oxford schema, right eye; L-Ox, corneal grading staining done by Oxford schema, left eye.

FTBUT. Furthermore, a positive correlation was found between right eye FTBUT and OSDI score. This suggests that PFH may allow the tear film to thicken, thus improving the tear-break-up time and, as a result, providing relief from DED symptoms. However, this may not be the only mechanism of action of the drug. Four patients had a negative change in CPTFT, but still showed improvement of FTBUT and OSDI. This implies that PFH influences not only the quantity but also changes the quality of the tear film. Future research could be extended to other parameters such as tear film osmolarity, matrix metalloproteinase level, meibography, and thermography of the ocular surface. $^{21-24}$

In Zhuang et al's study, mean precorneal tear film thickness was $24.7 \pm 3.9 \mu \mathrm{m}$ in normal eyes and $22.2 \pm 4.5 \mu \mathrm{m}$ in dry eyes. ${ }^{15}$ Another study confirmed that tear film thickness in dry eyes $(21.1 \pm 2.0 \mu \mathrm{m})$ was significantly smaller than that in normal eyes $(37.6 \pm 2.0 \mu \mathrm{m} ; \mathrm{p}<0.01) .{ }^{16}$ As in the present study, some DED patients had a CPTFT $\geq 24 \mu \mathrm{m}$ on Day 0, two groups were created, according to patient CPTFT on Day 0 (CPTFT $<24 \mu \mathrm{m}$ vs CPTFT $\geq 24 \mu \mathrm{m}$ ), when measuring the percentage of change in CPTFT. The percentage of change in CPTFT was the most significant in the group with a CPTFT $<24 \mu \mathrm{m}$ on Day 0 . In contrast, in patients with a CPTFT $\geq 24 \mu \mathrm{m}$ on Day 0 , the CPTFT either stayed unchanged or decreased. Although based on a small sample, it seems that in patients with a CPTFT close to the upper range limit for dry eye, further thickening of the tear film was not mandatory for improvement of the other parameters. ${ }^{15,16}$ Moreover, in patients with DED, the surface tension (ST) of the tear film is greater than that observed in patients with healthy eyes. ${ }^{25}$ In addition, ST is a logarithmic function proportional to the tear film thickness. ${ }^{26}$ Thus, an increase in tear film thickness above the upper limit for dry eye may lead to an increase in ST and, subsequently, to further tear film instability detected through a negative correlation between ST and non-invasive break-up time. ${ }^{25}$

The study showed an overall improvement in FTBUT of $33.33 \%$.

Tear film stability and symptoms are the most important parameters used in the diagnosis and management of $\mathrm{DED}^{22}$ In the present study, significant improvement in CPTFT, FTBUT, and OSDI was observed in a large number of patients, suggesting that PFH acted on the most important DED factors. The Schirmer test and vital staining of the surface are not mandatory for making a DED diagnosis. ${ }^{27}$ These 2 factors may be considered inadequate for diagnosing DED as supported by the fact that their lack of improvement did not result in a subjective improvement. Although the Schirmer test had the highest individual percentage of positive change among all the tested outcomes, suggesting a stabilizing effect of PFH on the tear film, it did not yield adequate symptom amelioration in these patients. ${ }^{5}$

Study results further confirmed that a continued use of PFH for 15 days improves OSDI, as reported by Kallab et al. ${ }^{13}$ In our study, OSDI had improved in all but one patient, for whom only 2 parameters had improved: the right eye Schirmer test and the left eye Oxford grading. This suggests that subjective worsening was reported only when most of the parameters failed to improve. Considering OSDI as a function of both eyes and using OSDI for grading DED severity, this questionnaire based on patient subjective answers, although evidencing a significant improvement with PFH, did not correlate with any of the objective parameters, and as such should not be grounds for determining DED, its diagnosis, or even its severity. 
The patterns of precorneal tear film pachymetry maps observed in the present study are in agreement with study results showing that the vast majority of eyes with DED had pachymetry pattern 1 ; however, results were statistically significant. ${ }^{15}$ In the present study group, the lack of a control group with normal eyes prevented calculating statistical significance. As a result, without enough tear film pachymetry studies involving a large enough number of patients; the focus should be on measuring the central zone of the precorneal tear film thickness when evaluating DED.

Again, our study confirmed the findings of Kallab et al: PFH does not impact on IOP and it is safe to use. ${ }^{13}$

As there was no significant correlation between the eyes' FTBUTs and between the eyes Schirmer tests, and as vital staining showed no significant change with $\mathrm{PFH}$, the CPTFT, with its significant percentage of change and correlation between the eyes, these parameters could become the most important elements for diagnosis, assessment of the severity, and of the management of DED.

DED is a chronic disease. Thus, treatment is long-lasting and should be personalized since it has to be adapted to the different clinical conditions observed along the course of the disease. ${ }^{28}$ Since DED treatment is frequently unable to provide fast and complete relief from symptoms, corticosteroids may be helpful to provide a quick relief. Moreover, empathy with patients and willingness to explain to them the natural history of the disease are mandatory to improve patients' compliance. Furthermore, patients should be instructed about the possible need to increase the frequency and/or change the type of treatment according to the fluctuation of symptoms, following a preplanned rescue regimen. ${ }^{28}$

Limitations of this study consist of its retrospective setting with no reference group and its small sample size. Furthermore, as DED is a chronic condition, a longer follow-up time would have been needed to evaluate improvement stability.

In conclusion, this retrospective data review confirms that topical preservative-free hydrocortisone $3.35 \mathrm{mg} / \mathrm{mL}$ twice daily for 2 weeks significantly improves precorneal tear film thickness, resulting in a significant decrease in subjective symptoms associated to dry eye, as measured with the OSDI.

\section{Acknowledgments}

The authors acknowledge Karl Patrick Göritz, SMWS, Scientific and Medical Writing Services, France for his medical writing assistance.

\section{Funding}

Study treatment was supplied by Laboratoires Théa, France.

\section{Disclosure}

The authors report no conflicts of interest in this work.

\section{References}

1. Craig JP, Nelson JD, Azar DT, et al. TFOS DEWS II report executive summary. Ocul Surf. 2017;15(4):802-812. doi:10.1016/j.jtos.2017. 08.003

2. Baudouin C, Irkec M, Messmer EM, et al. Clinical impact of inflammation in dry eye disease: proceedings of the ODISSEY group meeting. Acta Ophthalmol. 2018;96(2):111-119. doi:10.1111/ aos. 13436

3. Bron AJ, Yokoi N, Gafney E, Tiffany JM. Predicted phenotypes of dry eye: proposed consequences of its natural history. Ocul Surf. 2009;7(2):78-92. doi:10.1016/S1542-0124(12)70299-9

4. Lambiase A, Micera A, Sacchetti M, Cortes M, Mantelli F, Bonini S. Alterations of tear neuromediators in dry eye disease. Arch Ophthalmol. 2011;129(8):981-986. doi:10.1001/archophthalmol.2011.200

5. Willcox MDP, Argüeso P, Georgiev GA, et al. TFOS DEWS II tear film report. Ocul Surf. 2017;15(3):366-403. doi:10.1016/j.jtos.2017.03.006

6. Zhang X, Qu Y, He X, et al. Dry eye management: targeting the ocular surface microenvironment. Int J Mol Sci. 2017;18(7):1398. doi:10.3390/ijms 18071398

7. Messmer EM. The pathophysiology, diagnosis, and treatment of dry eye disease. Dtsch Arztebl Int. 2015;112(5):71-81;quiz 2.

8. Cutolo CA, Barabino S, Bonzano C, Traverso CE. The use of topical corticosteroids for treatment of dry eye syndrome. Ocul Immunol Inflamm. 2019;27(2):266-275. doi:10.1080/09273948.2017.1341988

9. Haber SL, Benson V, Buckway CJ, Gonzales JM, Romanet D, Scholes B. Lifitegrast: a novel drug for patients with dry eye disease. Ther Adv Ophthalmol. 2019;11:2515841419870366. doi:10.1177/ 2515841419870366

10. Hamard H, Schmitt C, Plazonnet B, Le Douarec JS. Study of the ocular penetration of dexamethasone. [Etude de la pénétration oculaire de la déxamethasone.] In: Demailly P, Hamard H, Luton JP, editors. Oeil et cortisone. Paris: Masson; 1975:33-84.

11. McGhee CN. Pharmacokinetics of ophthalmic corticosteroids. $\mathrm{Br} J$ Ophthalmol. 1992;76(11):681-684. doi:10.1136/bjo.76.11.681

12. Razeghinejad MR, Katz LJ. Steroid-induced iatrogenic glaucoma. Ophthalmic Res. 2012;47(2):66-80. doi:10.1159/000328630

13. Kallab M, Szegedi S, Hommer N, et al. Topical low dose preservative-free hydrocortisone reduces signs and symptoms in patients with chronic dry eye: a randomized clinical trial. Adv Ther. 2020;37 (1):329-341. doi:10.1007/s12325-019-01137-8

14. Schiffman RM, Christianson MD, Jacobsen G, Hirsch JD, Reis BL. Reliability and validity of the ocular surface disease index. Arch Ophthalmol. 2000;118(5):615-621.

15. Zhuang H, Zhou X, Xu J. A novel method for pachymetry mapping of human precorneal tear film using Pentacam with fluorescein. Invest Ophthalmol Vis Sci. 2010;51(1):156-159. doi:10.1167/iovs.08-3265

16. Kang NH, Park YG, Jun RM. Measurement of Precorneal tear film using scheimpflug camera and relationship with parameters for dry eye. J Korean Ophthalmol Soc. 2015;56(11):1699-1705. doi:10. 3341/jkos.2015.56.11.1699

17. Fariselli C, Giannaccare G, Fresina M, Versura P. Trehalose/hyaluronate eyedrop effects on ocular surface inflammatory markers and mucin expression in dry eye patients. Clin Ophthalmol. 2018;12:1293-1300. doi:10.2147/OPTH.S174290 
18. Mediately. Softacort 3,35 $\mathrm{mg} / \mathrm{ml}$ kapi za oko, otopina u jednodoznom spremniku. Available from: https://mediately.co/hr/drugs/ 98sHG12OKofkQVVLgKqws8me2mX/softacort-3-35-mg-ml-kapiza-oko-otopina-u-jednodoznom-spremniku. [Serbian]. Accessed August 15, 2020.

19. Sullivan DA, Rocha EM, Aragona P, et al. TFOS DEWS II sex, gender, and hormones report. Ocul Surf. 2017;15(3):284-333. doi:10.1016/j.jtos.2017.04.001

20. Farrand KF, Fridman M, Stillman IO, Schaumberg DA. Prevalence of diagnosed dry eye disease in the United States among adults aged 18 years and older. Am J Ophthalmol. 2017;182:90-98.

21. Su T-Y, Ho W-T, Chiang S-C, Lu C-Y, Chiang HK, Chang S-W. Infrared thermography in the evaluation of meibomian gland dysfunction. J Formos Med Assoc. 2017;116(7):554-559. doi:10.1016/j. jfma.2016.09.012

22. Lemp MA, Bron AJ, Baudouin C, et al. Tear osmolarity in the diagnosis and management of dry eye disease. Am J Ophthalmol. 2011;151(5):792-8e1. doi:10.1016/j.ajo.2010.10.032

23. Messmer EM, von Lindenfels V, Garbe A, Kampik A. Matrix metalloproteinase 9 testing in dry eye disease using a commercially available point-of-care immunoassay. Ophthalmology. 2016;123(11):2300-2308. doi:10.1016/j.ophtha.2016.07.028
24. Arita R. Meibography: a Japanese perspective. Invest Ophthalmol Vis Sci. 2018;59(14):DES48-DES55. doi:10.1167/iovs.17-23631

25. Tiffany JM, Winter N, Bliss G. Tear film stability and tear surface tension. Curr Eye Res. 1989;8(5):507-515. doi:10.3109/0271368 8909000031

26. Tiffany JM. Surface tension in tears. Arch Soc Esp Oftalmol. 2006;81 (7):363-366.

27. Tsubota K. Short tear film breakup time-type dry eye. Invest Ophthalmol Vis Sci. 2018;59(14):DES64-DES70. doi:10.1167/ iovs. 17-23746

28. Aragona P, Giannaccare G, Mencucci R, Rubino P, Cantera E, Rolando M. Modern approach to the treatment of dry eye, a complex multifactorial disease: a P.I.C.A.S.S.O. board review. $\mathrm{Br} J$ Ophthalmol. 2020:bjophthalmol-2019-315747. doi:10.1136/bjophthalmol-2019315747
Clinical Ophthalmology

\section{Publish your work in this journal}

Clinical Ophthalmology is an international, peer-reviewed journal covering all subspecialties within ophthalmology. Key topics include: Optometry; Visual science; Pharmacology and drug therapy in eye diseases; Basic Sciences; Primary and Secondary eye care; Patient Safety and Quality of Care Improvements. This journal is indexed on PubMed
Dovepress

Central and CAS, and is the official journal of The Society of Clinical Ophthalmology (SCO). The manuscript management system is completely online and includes a very quick and fair peer-review system, which is all easy to use. Visit http://www.dovepress.com/ testimonials.php to read real quotes from published authors. 